\title{
A plasma formation in pulsed coaxial gun at continuously filling regime
}

\author{
A. M. Zhukeshov, A. U. Amrenova, A. T. Gabdullina, B. M. Ibraev \\ Science Research Institute of Experimental and Theoretical Physics of Kazakh National University named after al-Farabi, Almaty, \\ Kazakhstan
}

\section{Email address:}

zhukeshov@physics.kz(A. M. Zhukeshov)

\section{To cite this article:}

A. M. Zhukeshov, A. U. Amrenova, A.T. Gabdullina, B. M. Ibraev. A Plasma Formation in Pulsed Coaxial Gun at Continuously Filling Regime. American Journal of Physics and Applications Vol. 1, No. 1, 2013, pp. 5-10. doi: 10.11648/j.ajpa.20130101.12

\begin{abstract}
In work presented the results of researches of plasma flow formation and propagate in the electrodynamics pulsed gun. Experimental researches carried out on the basis of pulse plasma accelerator CPU-30 with a coaxial symmetric geometry electrodes. It was shown, that structure of the flow significantly depends on initial operating pressure of working gas, and specify for "continuously filled" regime. The data on dynamics of plasma acceleration, obtained in previous studies was refined. The role of plasma internal electric field and acceleration peculiarities on differ pressure of initial gas are discuss.
\end{abstract}

Keywords: Plasma, Accelerator, Diagnostics, Magnet Field, Current

\section{Introduction}

Plasma flows generated by high voltage pulsed power accelerators, are widely used in physical and technological facilities. Despite the rather simplicity of construction of the device, dynamics formed in the plasma accelerator channel complicated way depends on the conditions of experiment and significantly from the geometry of the system. Detailed studies of the work of pulse plasma accelerators, carried out in $[1,2]$ have shown that for these accelerators there is a «critical» value of the current $J_{c r}$, when it reaches a sharp growth of discharge voltage and there is a change of the form of the VAC curve. In the accelerator generated oscillations of large amplitude. The authors connected this result to the influence of the Hall Effect in the flow of plasma across the magnetic field, leading to the emergence of the longitudinal component of the electric field, because of what is changing the structure of the discharge. A.I. Morozov on the basis of the analysis of the acceleration of a plasma in a magnetic field showed that realization of Hall Effect is possible at a sufficiently high density of the gas, at condition of quasi-neutral plasma [3]. Further experiments conducted on different accelerators [4, 5] show that the Hall Effect influence leads to the compression of the plasma in the side of one of electrodes and focus formation. Using a probe procedure, we established some features of the formation of flow in the pulse plasma accelerator CPA-30 [6]. Found that the flow consists of electronic and ionic components, but the phenomenon shown only at constant initial pressure of gas before shutting.

It is interest to connection the research of work of plasma accelerator on "continuously filled" regime. In this mode, the working gas fills the working chamber with a given initial pressure, which provides the ability to vary wide change the density of the plasma formed [8]. This mode allows receiving ion-plasma flows of a different composition, which is perspective for various technical and technological applications of plasma accelerators [8-12]. In this work carried out the diagnostics of plasma flow, formed at various initial pressure and voltage on the pulse plasma accelerator CPU-30. Detailed study of flow formation in acceleration canal by using of miniature magnet probe and coils.

\section{Experimental Setup}

Designed in SRIETP coaxial accelerator CPA-30 is used both for testing of developed diagnostic techniques, as well as for materials surface modification. Accelerator has a coaxial cylindrical electrodes made of copper length of 45 $\mathrm{cm}$, the diameter of the outer electrode of $9 \mathrm{~cm}$, inner $-3 \mathrm{~cm}$. Supply system consists of 25 capacitors with an operating voltage up $30 \mathrm{kV}$, the total capacity is $75 \mu \mathrm{F}$. Working gas is air. Used mode of operation of the accelerator at a constant pressure inside the discharge chamber in the range $(0,05-5)$ $\pm 20 \%$ Torr. Before each discharge chamber was evacuated 
to a pressure below $10^{-3}$ Torr. After that, depending on the task, the chamber filling gas to need pressure. The schematic diagram of setup shown in figure 1 .

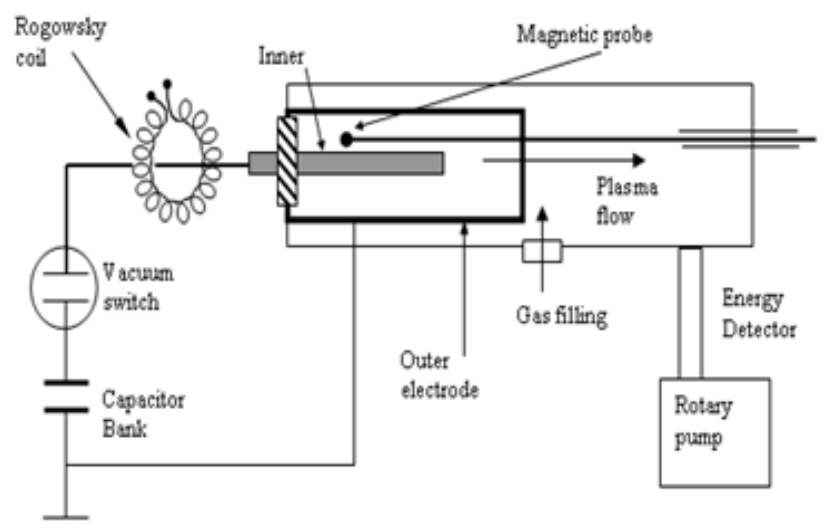

Figure 1. The experimental installation scheme.

The distribution of the magnetic field and the current was measured with magnetic probes and Rogowski belt. For the study used coils with a different number of turns, folded in the form of rings and installed outside the discharge power cables and inside the accelerator in a plane perpendicular to the direction of the plasma flow. Thus, within the accelerator is measured by the current passing through the cross-section of a ring. In the case of the outer belt of the current corresponds to discharge current of the capacitor, and in the case of an internal - current in the plasma. The external belt was made as the solenoid by a diameter of $4 \mathrm{~mm}$ and length $60 \mathrm{~cm}$, quantity of coils 2450 . The solenoid closes on small resistance $0,5 \mathrm{Ohm}$. To research of currents inside the accelerator applied a belt with quantity of coils 528 and length $30 \mathrm{~cm}$.

The tiny magnetic probe was applied to researches. The magnetic probe represented 5 coils of a wire by a diameter of $0,12 \mathrm{~mm}$ reeled - up on a skeleton by a diameter of $1,5 \mathrm{~mm}$. Strengthened at the end of the coil tightly interwoven wire that was placed in a quartz tube with a diameter of $4 \mathrm{~mm}$ and a length of $60 \mathrm{~cm}$. The equivalent area of the coil probe $n S$ is equal $0,26 \mathrm{~cm}^{2}$, and the inductance $L$ makes $1,0 \mu \mathrm{Hn}$. Thus the constant time $L / R_{0}$ about $2 \mathrm{~ns}$, that is very short for considered experiment. As the time, during which the changes of a magnetic field were considered, was about 1 iên, the passive integrating chain with $\mathrm{RC}=50 \mathrm{Ohm} \cdot 0,5 \mu \mathrm{F}$ $=2,5 \mu$ s was used. From here sensitivity of magnet probe

$$
\frac{V}{B}=\frac{n S}{R C}=200 \mu V / G
$$

This sensitivity has provided a direct connection to the oscilloscope without using additional amplifiers with an acceptable signal / noise ratio. In spite of the temperature in pulsed plasma accelerators is high $\left(\sim 10^{4} \mathrm{~K}\right)$, the time of exposure is small enough, so that the metal probes can be used here without the risk of melting.

To measure the high voltages used a combined resistive and capacitive voltage divider with division ratio of 1:10,000. The signals were studied using a digital oscilloscope with a bandwidth of $100 \mathrm{MHz}$.

\section{Experimental Results}

\subsection{The Discharge Current and Voltage}

Typical current waveforms at pressures of 0.1 and 0.05 Torr is shown in Figure 2. The discharge current in the accelerator is periodic damped signal. The number of half period is almost independent of the initial pressure in the chamber. The period of the current increases slightly by 1-2 $\mu \mathrm{s}$, the current amplitude decreases exponentially with a decrement of $\sim 10^{5}$. The presence of rapidly damped oscillation at the beginning of the sweep can be attributed to the reflection signal from the cable ends, which is typical for such measurements. Figure 2 (bottom) also shows the voltage waveforms at this pressure. Attention is drawn to the following features of these curves. First, the line voltage is strongly indented, which is caused by impulse noise. At the current curves in the initial moment of time, you can also notice the presence of rapid oscillations, but since the Rogowski coil has a large self-inductance irregularity is manifested not so much. In addition, at the beginning of the discharge a current increases very rapidly, reaching $500 \mathrm{kA}$ for a time of $3 \mu \mathrm{s}$. In this case, the rate of current rise will be $1.7 \cdot 10^{11} \mathrm{~A} / \mathrm{sec}$. At second, the voltage in the first quarter of the discharge is greater than the initial value and then decreases. Such behavior, apparently, to the effect described in the literature as anomalous plasma resistance at the initial moment of the discharge [13]. In this case, the total resistance of the plasma dominates its ohmic resistance. As a result, the voltage across the discharge gap increases.

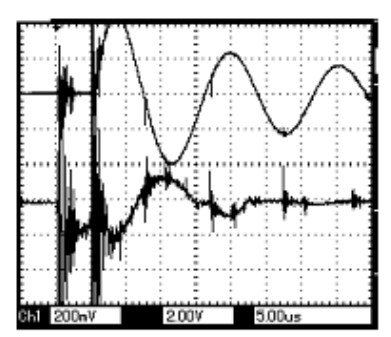

(a)

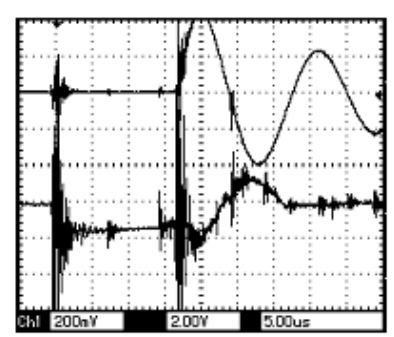

(b)
Figure 2. Discharge current and voltage signals measured at 0,1 (a) and 0,05 (b) Torr.

The detailed measurements of magnitude of signals are shown, that a value of the discharge current in the range $150-500 \mathrm{kA}$ are linear, and almost the same at any pressure within the discharge chamber of the accelerator. The volt ampere curve of discharge at different pressure shown in figure 3. The dependence of discharge current value from voltage is linearity for any pressures in considered experiment. The magnitude of current not strongly depends on pressure at constant voltage. 


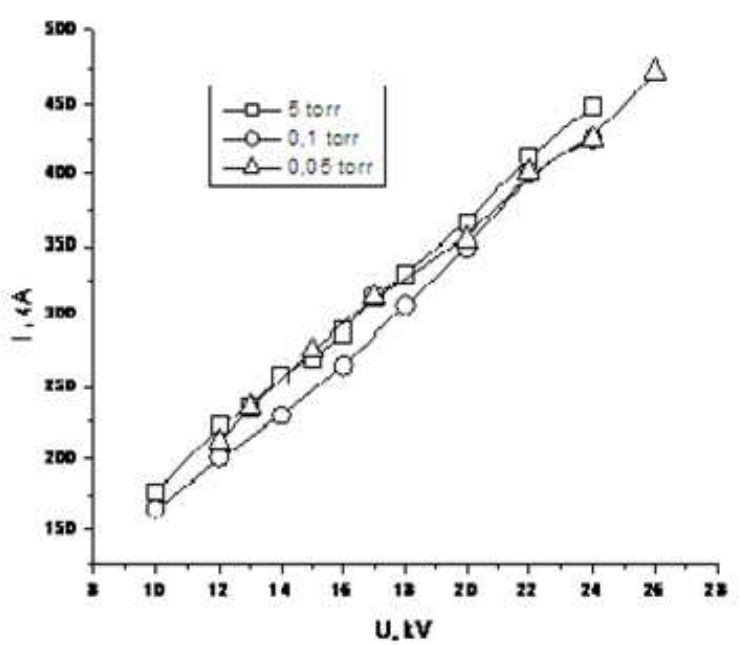

Figure 3. The volt - ampere curve of discharge.

\subsection{The Magnetic Field and Plasma Velocity}

The magnetic field distribution inside the interelectrode space was obtained with the magnetic probe. Signals from the magnetic probe, taken at a distance of $26 \mathrm{~cm}$ from the muzzle of the outer electrode are shown in Figure 4. By lowering the sensor detects of high-frequency oscillations of the magnetic field. These oscillations are a broadband signal in the range of $5.2 \mathrm{MHz}$, to get rid of vibrations can be achieved by integrating circuit with the time constant for more than $3 \mathrm{~ms}$.

With the help of magnetic probes were obtained by waveform $\mathrm{B}(\mathrm{t})$ for different positions of the probe in the middle between the cylindrical electrodes at a distance of 1 $\mathrm{cm}$ to $40 \mathrm{~cm}$ from the edge of the outer electrode. Discharges made under the same conditions: the voltage on the capacitor $20 \mathrm{kV}$ and a pressure of 0.1 and 1 Torr. The results showed good reproducibility of the signal from discharge to discharge.

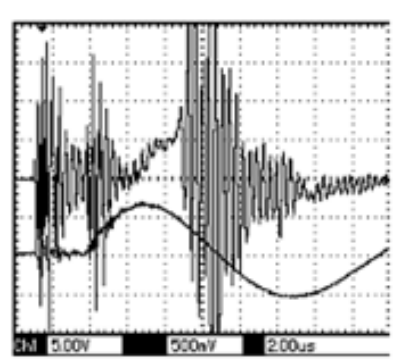

(a)

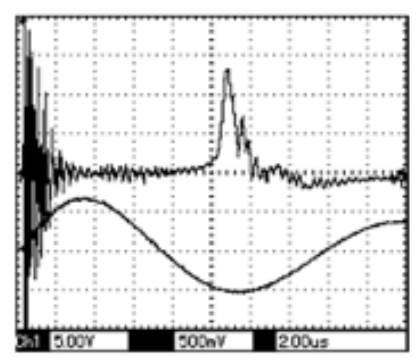

(b)
Figure 4. The Signals from magnetic probe measured at 0,1 (a) and 1 (b) Torr.

Next, we do analyzing a series of waveforms of the magnetic field along the axial axis at various distances from the edge of the outer electrode.

The magnetic field on plasma accelerator moves forward in the axial axis of the exit of the electrode. The average velocity of the front signal was $2.5 \mathrm{~cm} / \mu \mathrm{sec}$. Rate of rise of the front is about $1 \mu \mathrm{s}$, so the thickness of the current sheet, on which passes the discharge current of $2-3 \mathrm{~cm}$ at a pressure of 1 Torr. The average front velocity signal was $2.3 \mathrm{~cm} / \mu \mathrm{sec}$, which is almost equal to the rate at 0.1 Torr. Rate of rise of the front was about 2 microseconds, so the thickness of the current sheet, on which is the discharge current, was $4-5 \mathrm{~cm}$ Thus, the speed of the current layer is weakly dependent on pressure. Of interest to determine the velocity by another way, namely by two belts installed at a distance from each other. Results of experimental measurements are shown in Figure 5.

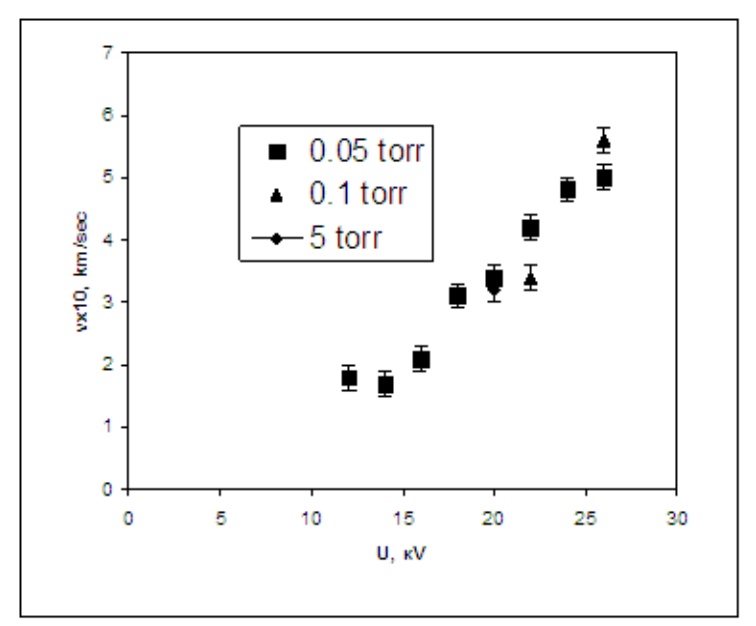

Figure 5. The average velocity of plasma flow at different pressures.

In accordance with Figure 5, the dependence of the velocity from voltage of non-linear. The maximum flow rate at a voltage of $27 \mathrm{kV}$ was $(5,6 \pm 0,3) \mathrm{cm} / \mu \mathrm{sec}$. In addition, there is also no noticeable difference in flow speed at different pressure. But, the

Next, Figure 6 shows a plot of the amplitude of the magnetic field in the interelectrode space. The amplitude of the magnetic field along the electrodes behaves uniquely. At pressure value 1 Torr, it is the same order of magnitude at the beginning and at the outlet (on the graph on the left) electrode. Follow, at 0.1 Torr, amplitude reduced from 30 kGauss to near zero.

\subsection{The Plasma Focusing and PRopagate}

Above was investigated area inside coaxial electrodes, but not less important is the compression zone, located by slice electrodes. In the presence of currents of fans in this area, you can focus the power line current.

Information about the structure of the plasma in the area adjacent to the edge of the electrode, given current measurements obtained using miniature Rogowski belt. The coil located on the three lines from edge of the electrode to the middle of the axial axis. The distance from the end of the center electrode changed in one $\mathrm{cm}$. There was thus obtained a series of waveforms in which the current density is counted and building the lines of equal current. The measurements 
showed significantly different picture of the current distribution at different initial pressure.

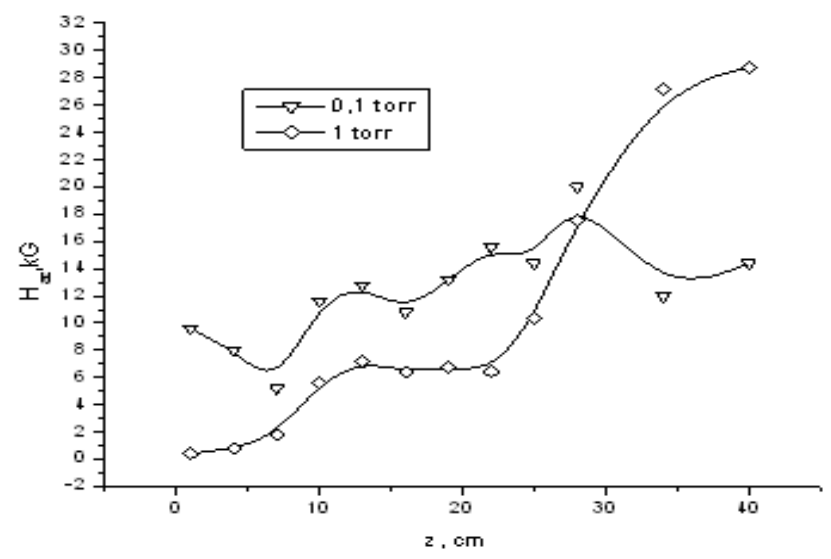

Figure 6. The magnetic field magnitude distribution

For example, in figure 7 shows the current waveform on the axis at a distance of $1 \mathrm{~cm}$ from the end of the inner electrode, but at two different pressures. It is seen that at 0.1 Torr current signal appears after a period after the discharge current (see Figure 4.23). And at 1 Torr, almost immediately, but opposite amplitude

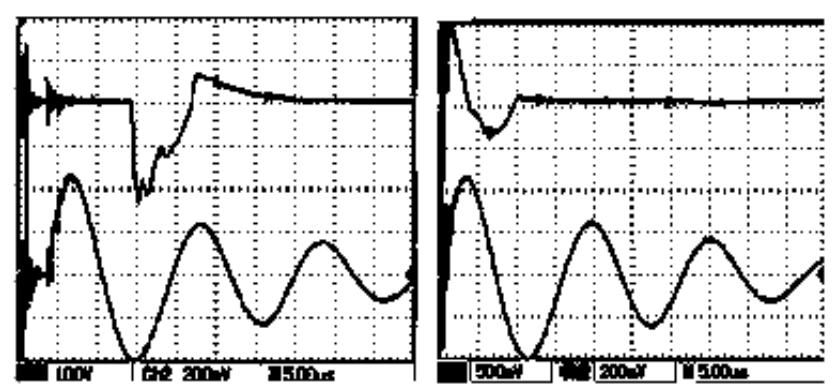

Figure 7. The plasma (up) and discharge current (bottom) waveforms at 0,1 and 1 Torr.

Further, in accordance with figure 7 , with the probe is moved away from the coaxial axis to the edge of electrode, the signal changes its polarity. Since the cross section of the probe is sufficiently small, it indicates that the current (more precisely, the axial component of the current) changes its direction.
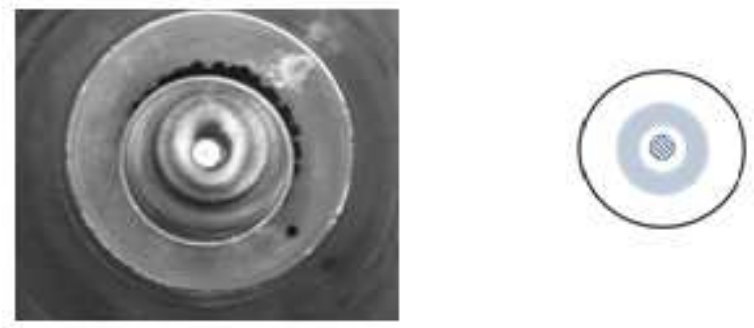

Figure 8. The erosion of CPA-30 electrodes and schematic diagram of this process on coaxial system
That is, the flow lines go from the end of the center electrode and come to the edge of the outer electrode, making the semi-circle. Picture of the formation of currents in the area of focus will be clearer if we look at the photos of the accelerator electrode system shown in Figure 8. So, the area side of the central electrode (cylinder surface) contains minor traces of erosion. At the same time, the ends of the central electrode (start and end) are melted, indicating the presence of large electron currents at these surfaces. The sputtered areas marked on the diagram at right.

Based on the combination of these and other experimental data is drawled the scheme of flow distribution in the accelerator CPA-30, shown in Figure 9.

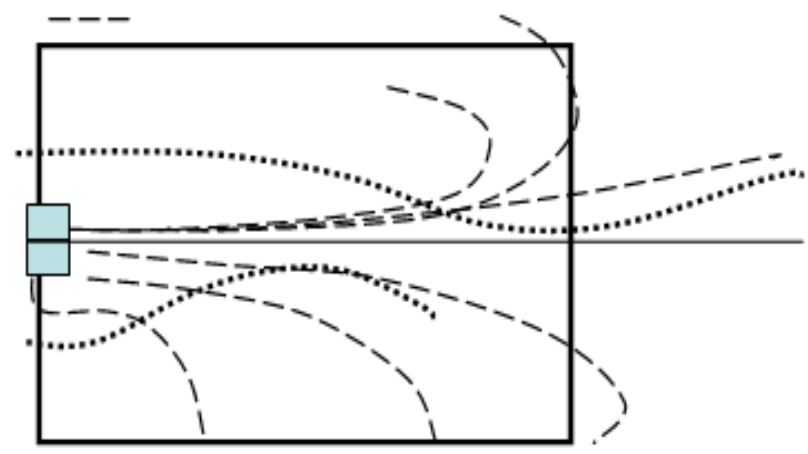

Figure 9. The flow distribution schematically in plasma accelerator CPA-30

This diagram shows at the map of the current line (electrons- drop line, an the ions -point line) at a pressure of 0.1 Torr (top) and at the bottom - with a pressure of 1 Torr. Since ions are heavy particles, their movement along the electrodes is substantially a straight line. However, that because of the current removal of electrons ions is forced to shrink to the axis of the system, since there is a large negative charge density. Because of this, at some distance from the end of the central electrode is formed should focus.

The formation of plasma focus at distance about $10 \mathrm{~cm}$ from muzzle of central electrode, was shown early by our research[14].

\section{Discuss}

Thus, in this study using magnetic probes investigated the magnetic field distribution along the electrodes of the accelerator CPA. It is established that the magnetic field moves in a direction to the outlet of the electrodes. The average flow velosity is practically independent of the initial gas pressure in the chamber of the accelerator, at least within the limits of 0.1-1 Torr. This result can be explained by the fact that according to Spitzer formula, charged particle concentration in plasma is determined by the degree of ionization. In this case, at sufficiently high voltage to the discharge, achieved full ionization of the gas, and in this case, the discharge current and flow rate does not depend on pressure. 
Voltage dependence of the rate of non-linear and is described by a power law. These results are evidence that this accelerator main force of the movement is electrodynamics force [11], since in this case, the flow rate.

$$
v \sim J^{2} t^{n}
$$

Analysis of experimental data suggests the magnitude of the initial pressure in the chamber has a significant influence on the dynamics of the magnetic field inside the electrode space. At lower pressures the electromagnetic energy is proportional to the magnetic field is realized in the initial stage of acceleration. High pressure is evenly distributed along the length of the accelerator. In this case, the acceleration of the magnetic force is the Ampere's. At the same time, at a pressure below 0.1 Torr in the accelerator, there are significant oscillations in the magnetic field, but they tend to decay with increasing pressure. We can assume that in this case is formed in the accelerator instability associated with anomalous plasma resistance, the formation of structures such as filaments [13], which leads to an increase in voltage across the electrodes at the beginning of the discharge. Further development of the instability leads to a buildup of large amplitude oscillations. However, the appearance of oscillations does not fit into the framework of electrodynamic acceleration model, but perhaps an explanation for the effect of Hall accelerators. However, as shown A. Morosov, the Hall Effect manifests itself at a high enough density of the gas, when the plasma has been in quazy- neutral state [14]. In this case the acceleration depends of the longitudinal electric field. In our case, we observe a significant acceleration at pressures below 0.1 Torr, when the Hall Effect should reduce. Therefore, the explanation of a significant acceleration of the plasma at very low pressures requires a different approach. We believe that the acceleration of the plasma in low density plasma could occur as a result of the action of the electric field of the space charge of electrons. At low density plasma the mean free path of electrons is comparable to the size of the electrodes and possible large-amplitude fluctuations that we see in the oscillograms (picture 4). Erosion of the end and the beginning of electrodes indicates that its way to complete on these surfaces (depending on the polarity of the central electrode at the moment). The Ions have been magnetized by field, they move only along the electrodes and exposed.

Thus, this study demonstrates that the mechanism of plasma acceleration is characterized by varying the initial pressure in the chamber of the accelerator. At high pressure acceleration possible by impact of Ampere force, and a low pressure of electric field in the plasma. However, such behavior of the plasma is only possible in the continuous mode, when the gas filling of the working chamber. It is a feature of this mode of operation, and can be used for obtain of very rapid plasma flow with high speed.

\section{References}

[1] Plasmennye uskoriteli [Ed. L.A. Arcimovich]. Moscow: Mashinostroenie, 1973, $312 \mathrm{p}$.

[2] Fizika i primenenie plasmennyh uskoriteley [Ed. A.I. Morosov].Minsk: Naukova dumka, 1974, 399 p.

[3] A.I. Morosov, A.P. Shubin. "K teorii electromagnitnyh processov pri nalichii effekta Holla", JETP, Vol.46, 1964, p. 710.

[4] Woong C.K., Chung K.H. and Byung H.C. "Characteristics of Hall effect plasma accelerator for industrial application", Rev. Sci. Instr., 1994, Vol 65(4), pp.. 1356-1358.

[5] K. F. Schoenberg, R. A. Gerwin at al. "Magnetohydrodynamic flow physics of magnetically nozzled plasma accelerators with applications to advanced manufacturing", Phys. of Plasmas, 1998 ,Vol. 5, Iss. 5, pp. 2090-2104.

[6] A. M. Zhukeshov, F. B Baimbetov, A. U. Amrenova. "Dynamics of Plasma Flow Formation in a Pulsed Accelerator Operating at a Constant Pressure", Tech. Phys. Lett., 2007, Vol. 33, No. 1, pp. 77-79.

[7] A.M. Zhukeshov. Plasma flow formation in a pulse plasma accelerator in continuos filling regime. Plasma Dev. Oper.17. (2009). P.73-81.

[8] V. I. Tereshin, A. N. Bandura, O. V. Byrka at al. "Repetitive Plasma Loads Typical for ITER Type-I ELMS: Simulation in QSPA Kh-50”, AIP Conf. Proce., January 15, 2006, Vol. 812, Issue 1, pp. 128-135.

[9] V.V. Chebotarev, I.E. Garkusha, A.M. Bovda at al. "Application of pulsed plasma accelerators for surface modification", Nukleonika, 2001, Vol. 46, pp. 27-30 .

[10] Z. Peng, H. Miao, W. Wang "Hard and wear-resistant titanium nitride films for ceramic cutting tools by pulsed high energy density plasma". Surf. Coat. Tech., 2003, Vol. 166 (2), pp. 183-188.

[11] Tereshin V.I., Bandura A.N., Byrka O.V. at al. "Surface Modification and Coatings Deposition under Plasma Streams Processing", Adv. Appl. Plasma Sci., 2003, Vol. 4, pp. 265-270.

[12] A. M.Zhukeshov, A.T. Gabdullina. "Vlianie rejimov obrabotki impulsnymi potokami plasmy na poverchnost stali io structuru i microtverdos”. Poverhnost, 2009. №11, pp. 95-101.

[13] A.G. Oreshko . "The domain model of anomaly resistance of plasmas", Voprosy Atomnoy Nauki Techniki, 2000,. Vol.1, pp. 17-21.

[14] A.I. Morosov. Vvedenie v plasmodynamiku. Moskov: Fizmatlit. 2007. 567 p. 